C. Oliver Hanemann, $\mathrm{MD}, \mathrm{PhD}$

Jaishri O. Blakeley, MD

Fabio P. Nunes, MD, MMSc

Kent Robertson, MD

Anat Stemmer-

Rachamimov, MD

Victor Mautner, MD

Andreas Kurtz, PhD

Michael Ferguson, MD

Brigitte C. Widemann, MD

D. Gareth Evans, MD

Rosalie Ferner, MD

Steven L. Carroll, MD, $\mathrm{PhD}$

Bruce Korf, MD, PhD

Pierre Wolkenstein, MD

Pamela Knight

Scott R. Plotkin, MD,

$\mathrm{PhD}$

For the REiNS

International

Collaboration

Correspondence to

Dr. Hanemann:

Oliver.Hanemann@plymouth.ac.uk

Supplemental data at Neurology.org

\section{Current status and recommendations for biomarkers and biobanking in neurofibromatosis}

\section{ABSTRACT}

Objective: Clinically validated biomarkers for neurofibromatosis 1 (NF1), neurofibromatosis 2 (NF2), and schwannomatosis (SWN) have not been identified to date. The biomarker working group's goals are to (1) define biomarker needs in NF1, NF2, and SWN; (2) summarize existing data on biomarkers in NF1, NF2, and SWN; (3) outline recommendations for sample collection and biomarker development; and (4) standardize sample collection and methodology protocols where possible to promote comparison between studies by publishing standard operating procedures (SOPs).

Methods: The biomarker group reviewed published data on biomarkers in NF1, NF2, and SWN and on biobanking efforts outside these diseases via literature search, defined the need for biomarkers in NF, and developed recommendations in a series of consensus meetings.

Results: We describe existing biomarkers in NF and report consensus recommendations for SOP and a minimal clinical dataset to accompany samples derived from patients with NF1, NF2, and SWN in decentralized biobanks.

Conclusions: These recommendations are intended to provide clinicians and researchers with a common set of guidelines to collect and store biospecimens and for establishment of biobanks for NF1, NF2, and SWN. Neurology ${ }^{\circledR}$ 2016;87 (Suppl 1):S40-S48

\section{GLOSSARY}

5-S-CD = 5-S-cysteinyldopa; ADM = adrenomedullin; MIA = melanoma inhibitory activity; $\mathbf{M P N S T}$ = malignant peripheral nerve sheath tumors; NF1 = neurofibromatosis 1; NF2 = neurofibromatosis 2; PNF = plexiform neurofibromas; REiNS = Response Evaluation in Neurofibromatosis and Schwannomatosis; $\mathbf{s A X L}=$ soluble growth factor receptor Axl; $\mathbf{S O P}=$ standard operating procedure; $\mathbf{S W N}=$ schwannomatosis.

The neurofibromatoses—neurofibromatosis 1 (NF1), neurofibromatosis 2 (NF2), and schwannomatosis (SWN) — are genetically distinct neurocutaneous syndromes that share many features. All 3 conditions demonstrate wide variability in disease manifestations, and are characterized by progressive, lifelong, and potentially life-threatening complications. Standard treatment is limited to surgery for most tumor manifestations. Given the unmet need for nonsurgical therapies, there have been $>20$ clinical trials performed between 1993 and 2014 for NF1 and NF2 with varying measures of response. Few studies have documented evidence of clinical efficacy for investigational agents. ${ }^{1}$ To date, no biomarker-driven trials have been performed in NF.

The Response Evaluation in Neurofibromatosis and Schwannomatosis (REiNS) International Collaboration was created in 2011 to define and develop the most informative, reliable, and meaningful endpoints for clinical trials for NF. The REiNS group is composed of several working groups. ${ }^{2}$ The biomarker working group, which includes neurologists, oncologists, geneticists,

\footnotetext{
From Plymouth University (C.O.H.), Peninsula Schools of Medicine and Dentistry, The Institute of Translational and Stratified Medicine, Plymouth, UK; Department of Neurology (J.O.B.), Johns Hopkins University Medical School, Baltimore, MD; Department of Pediatrics (F.P.N.) and Department of Pediatrics, School of Medicine (K.R., M.F.), Indiana University; Tailored Therapeutics (F.P.N.), Eli Lilly and Company, Indianapolis, IN; Department of Pathology (A.S.-R.), Neuro-oncology (S.R.P.), Massachusetts General Hospital, Boston; Neurologische Klinik (V.M.), Uniklinik Eppendorf, Hamburg; Berlin-Brandenburg Center for Regenerative Therapies (A.K.), Charité Universitätsmedizin Berlin, Germany; Seoul National University (A.K.), College of Veterinary Medicine and Research Institute for Veterinary Science, Republic of Korea; NCI (B.C.W.), Pediatric Oncology Branch, Bethesda, MD; Genomic Medicine (D.G.E.), University of Manchester, UK; National Neurofibromatosis Service (R.F.), Department of Neurology, Guy's and St. Thomas' NHS Foundation Trust, London UK; Department of Pathology and Laboratory Medicine (S.L.C.), Medical University of South Carolina, Charleston; and Heflin Center for Genomic Sciences (B.K.), University of Alabama at Birmingham; Dermatology (P.W.), GHU Henri Mondor, Paris, France; Children's Tumor Foundation (P.K.), New York. REiNS International Collaboration members are listed on the Neurology ${ }^{\circledR}$ Web site at Neurology.org. Go to Neurology.org for full disclosures. Funding information and disclosures deemed relevant by the authors, if any, are provided at the end of the article.
} 
pathologists, dermatologists, pediatricians, and basic scientists, has the goals to (1) define biomarker needs in NF1, NF2, and SWN; (2) summarize existing data on biomarkers in NF1, NF2, and SWN; (3) outline initial recommendations for sample collection and biomarker development; and (4) harmonize sample collection and processing protocols where possible to allow for data comparison between studies by publishing standard operating procedures (SOPs).

This article summarizes the progress toward these goals. The biomarker group has concentrated on biomarkers in blood, urine, and tissue. Imaging biomarkers are discussed separately by the REiNS imaging working group. ${ }^{3}$

METHODS The biomarker group first performed a literature search, and reviewed and summarized existing data on biomarkers in NF1, NF2, and SWN. The group then met during a series of meetings in collaboration with the Children's Tumor Foundation (1) to establish SOPs for sample collection of NF tissue specimens that facilitate data comparison between studies and (2) to develop a minimal clinical dataset that would accompany each sample. The working group anticipates that these recommendations will be updated periodically as new information on biomarkers becomes available. Detailed assay protocols will be available on the REiNS Web site (www.reinscollaboration.org).

RESULTS Biomarkers are used for early detection of disease or in disease classification (diagnostic biomarkers), in predicting response or adverse events (predictive biomarkers), in defining optimal drug dose (metabolic/pharmacodynamic biomarkers), or in forecasting progression or recurrence (outcome biomarkers). ${ }^{4}$ Previous natural history studies in NF1 and NF2 have clearly demonstrated a high degree of variability in disease phenotype and tumor behavior in these conditions. ${ }^{5}$ This variability introduces complexity into the identification of valid biomarkers for NF and schwannomatosis. For example, a biomarker could potentially correlate with the presence of the genetic syndrome (e.g., NF1, NF2, or schwannomatosis), of a specific tumor type (e.g., gastrointestinal stromal tumor in NF1), with a nontumor phenotype of NF (e.g., pain severity in schwannomatosis), with cumulative disease burden (e.g., whole body tumor burden), with disease progression (e.g., growth of plexiform neurofibroma in NF1), or with malignant transformation (e.g., malignant peripheral nerve sheath tumor from plexiform neurofibroma). Given the overlap in many of these phenotypes, validating these biomarkers for individual disease manifestations will require careful phenotyping of patients.

Diagnostic biomarkers in NF. Historically, NF1, NF2, and schwannomatosis have been diagnosed using established clinical criteria. Advances in molecular techniques over the last 2 decades have led to the availability of genetic diagnosis for these conditions. ${ }^{6.7}$ The sensitivity of genetic analysis for diagnosis of NF depends on the founder status and the clinical phenotype (segmental vs generalized) but ranges from $30 \%$ for sporadic schwannomatosis to $>95 \%$ for NF1. When a causative genetic alteration in the NF1, NF2, SMARCB1, or LZTR1 genes is identified, it can be used as diagnostic biomarker for related family members. In clinical practice, this information is used by reproductive endocrinologists for prenatal diagnosis or preimplantation genetic diagnosis and by genetic counselors for presymptomatic diagnosis of family members. However, additional diagnostic biomarkers will undoubtedly be useful to identify patients with specific disease features, such as plexiform neurofibromas or optic gliomas.

Outcome biomarkers in NF. Similarly, there is great variability in clinical outcomes for patients with NF. For example, while optic pathway glioma occurs in about $15 \%$ of individuals with NF1, only about $1 / 3$ of these tumors are symptomatic, and less will progress and require active treatment. ${ }^{8}$ Optic pathway gliomas will even spontaneously regress without treatment. This variability in outcome highlights the need to develop outcome biomarkers for NF disease manifestations. Outcome biomarkers provide evidence about the patient's disease outcome independent of any specific intervention. A validated outcome biomarker would be valuable to determine the long-term risk for dermal neurofibromas and malignant peripheral nerve sheath tumors in patients with NF1, for risk of complete deafness in patients with NF2, and for risk of severe chronic pain in patients with schwannomatosis.

Predictive biomarkers in NF. Over the last decade, there has been an explosion of clinical trials for NF-related tumors and conditions. The opportunity to study novel agents in NF opens the possibility of developing and incorporating biomarker studies into early clinical trials. To date, clinical trials run through the Neurofibromatosis Clinical Trials Consortium have included studies of pharmacodynamic biomarkers and predictive biomarkers. As drugs with activity against NF become available, these studies will provide valuable information about which patients are likely to require treatment in the future and which are likely to respond to therapy.

Existing data on biomarkers in NF1, NF2, and SWN. During development, biomarkers pass through a series of stages, including early research and development (exploratory biomarker), demonstration and validation (probable biomarker), characterization and qualification (known biomarker), and surrogacy (biomarker can substitute for clinical endpoint). For the neurofibromatoses, all 
Table 1 Exploratory biomarker studies published for neurofibromatosis 1 (NF1)

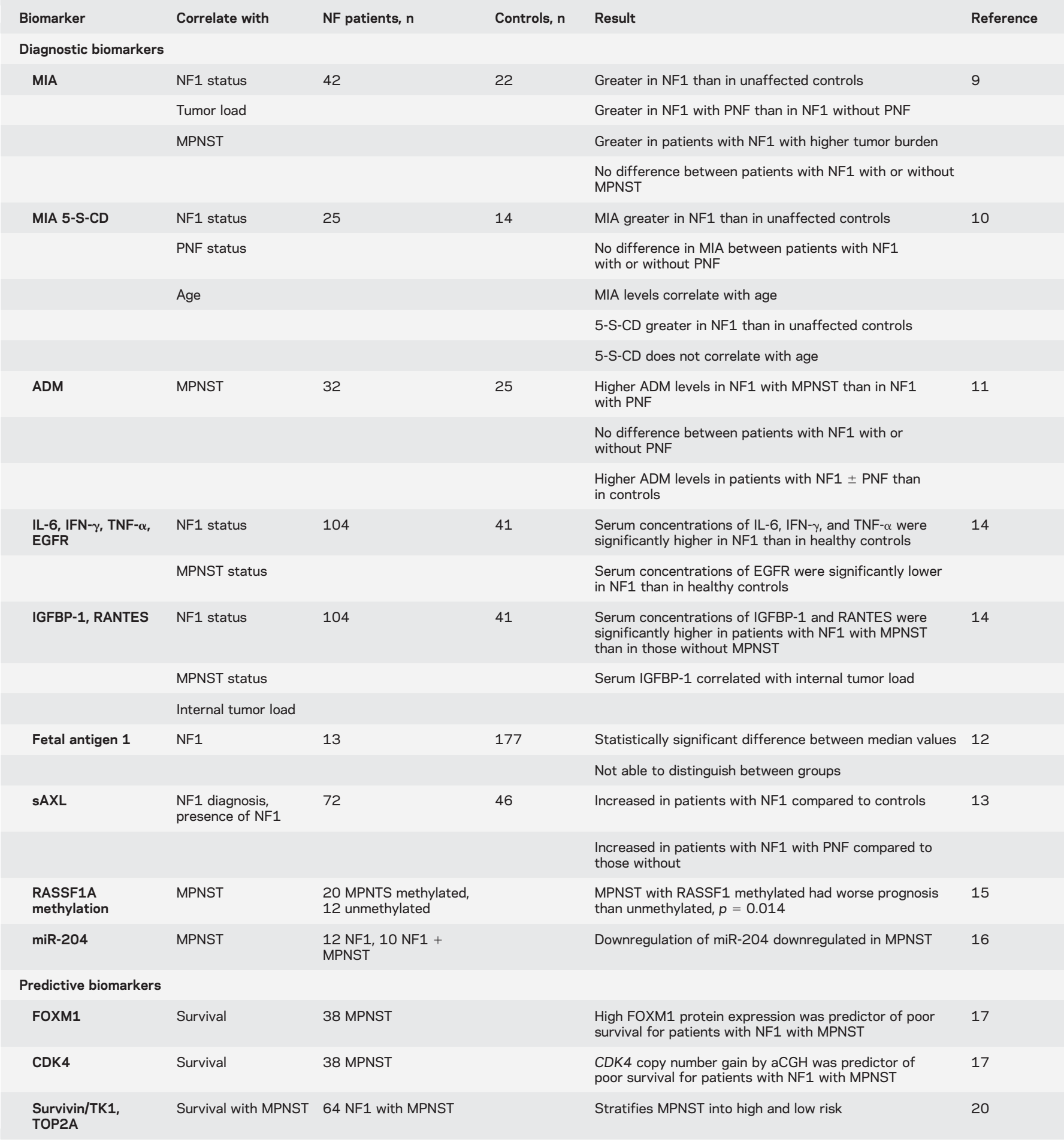

Abbreviations: 5-S-CD = 5-S-cysteinyldopa; aCGH = array comparative genomic hybridization; ADM = adrenomedullin; EGFR = epidermal growth factor receptor; IFN = interferon; IL = interleukin; MIA = melanoma inhibitory activity; miR = microRNA; MPNST = malignant peripheral nerve sheath tumors; $\mathrm{PNF}=$ plexiform neurofibromas; $\mathrm{sAXL}=$ soluble growth factor receptor $\mathrm{Axl} ; \mathrm{TNF}=$ tumor necrosis factor.

biomarkers published to date are considered exploratory; none has been validated to achieve probable biomarker status. Thus far, all published biomarker studies have been performed in the setting of NF1 (table 1); there are no published data on biomarkers in NF2 or schwannomatosis. These exploratory studies are all limited by a variety of technical issues such as limited statistical power, lack of an independent validation set, and lack of longitudinal data.

Diagnostic biomarkers in NF1.

- Melanoma inhibitory activity (MIA) - In a study of 42 patients with NF1 and 22 healthy controls, ${ }^{9}$ MIA serum levels were significantly 
greater in patients with NF1 $(15.2 \mathrm{ng} / \mathrm{mL})$ than in controls $(4.5 \mathrm{ng} / \mathrm{mL})$, in patients with NF1 with plexiform neurofibromas (PNF) than in those without PNF, in patients with NF1 with high numbers of cutaneous or subcutaneous neurofibromas than in those without such tumors, and in patients with NF1 with greater internal tumor load than in those with low internal tumor load. In contrast, serum levels of MIA were not significantly different between patients with NF1 with and without malignant peripheral nerve sheath tumors (MPNST). MIA as a biomarker for NF1 was also investigated by another group. ${ }^{10}$ This group added the analysis of the metabolite 5-S-cysteinyldopa (5-S-CD). They found concordant results with higher MIA levels in 25 patients with NF1 compared with 14 controls (13.1 vs $9.3 \mathrm{ng} / \mathrm{mL}$ ) but did not find significantly different levels between patients with NF1 with or without PNF (15.1 vs $12.0 \mathrm{ng} / \mathrm{mL}$, respectively). In their study, serum 5-S-CD levels were significantly higher in patients with NF1 than in controls and did not correlate with age. Hence, MIA does not appear to be a robust biomarker for distinguishing unaffected controls from patients with NF1 or between patients with NF1 with or without MPNST. Further work is needed to explore the utility of 5-S-CD to distinguish between groups.

- Adrenomedullin (ADM) — In a study of 32 patients with NF1 and 25 control patients, ${ }^{11}$ serum ADM levels were significantly greater in patients with NF1 with MPNST $(0.24 \mathrm{ng} / \mathrm{mL}, \mathrm{n}=5)$ than in patients with NF1 with PNF $(0.18 \mathrm{ng} / \mathrm{mL}$, $\mathrm{n}=17$ ); however, there was significant overlap in ADM levels between the cohorts. ADM levels were also significantly higher in patients with NF1 with or without MPNST $(0.18 \mathrm{ng} / \mathrm{mL}, \mathrm{n}=27)$ than in controls $(0.07 \mathrm{ng} / \mathrm{mL}, \mathrm{n}=25)$. No significant differences were noted between patients with NF1 with or without PNF. Although the data for $\mathrm{ADM}$ data are more promising than those for MIA, the current data do not indicate that $\mathrm{ADM}$ is a reliable biomarker in NF1 and further studies are warranted.

- Fetal antigen 1 -In a study of 13 adult patients with NF1 and 177 adult controls, ${ }^{12}$ the median fetal antigen 1 levels were significantly higher in the serum of patients with NF1 $(30.6 \mathrm{ng} / \mathrm{mL})$ than in control patients $(24.9 \mathrm{ng} / \mathrm{mL})$, although there was considerable overlap between the groups. $^{12}$

- Soluble growth factor receptor Axl (sAXL) In a study of 72 patients with NF1 and 46 healthy controls, serum levels of sAXL were significantly increased in patients with NF1 (23 ng/mL) compared with controls (16 ng/ $\mathrm{mL}$ ). Patients with plexiform neurofibromas had significantly higher levels of sAXL (26 ng/mL, $\mathrm{n}=36$ ) than patients with NF1 without plexiform neurofibromas (18 ng/mL, $\mathrm{n}=36$ ) although there was still some overlap between groups. ${ }^{13}$

- Systematic screen of multiple serum biomarkers ${ }^{14}$-The authors evaluated 104 patients with NF1 and 41 healthy controls and proposed 4 candidate biomarkers that were statistically significantly different in patients with NF1 vs unaffected individuals (interleukin-6, interferon- $\gamma$, epidermal growth factor receptor, tumor necrosis factor- $\alpha$ ) and 2 biomarker candidates that were statistically significantly different in patients with NF1 with and without MPNST (insulin-like growth factor binding protein 1 and RANTES).

- Danielsen et al. ${ }^{15}$ analyzed the promoter methylation of RASSF1A in primary tumor. The authors reported significantly different survival data for 32 patients with methylated or unmethylated $R A S S F 1 A$ at 5 years.

- MiRNA studies in NF1 and non-NF1 MPNSTs have been performed using cell lines and tissues. This study identified the downregulation of miR204 as a novel diagnostic biomarker and potential therapeutic target for patients with NF1 with MPNSTs. ${ }^{16}$

All the above-mentioned studies were aimed at identifying diagnostic biomarkers.

\section{Predictive and pharmacodynamic biomarkers.}

- Candidate gene alterations in tumor specimensUsing high-resolution array-based comparative genomic hybridization in 38 MPNSTs (23 with NF1 and 15 without NF1), the authors identified candidate gene alterations that were then validated at the DNA, RNA, and protein levels. ${ }^{17}$ Candidate genes in regions of copy number gain and associated (predicted) with poor survival included SOX5, NOL1, MLF2, FOXM1, FKBP1, CDK4, TSPAN31, ERBB2, MYC, and TP53. In multivariate analysis, FOXM1 protein expression and $C D K 4$ copy number gain were the most significant independent genomic biomarkers for poor survival in patients with MPNSTs.

- Aurora kinase A, which is important in mitotic spindle assembly and nonmitotic related cell differentiation, is a potential therapeutic target in MPNSTs. Recent studies in primary MPNSTs have demonstrated that pharmacologic aurora kinase inhibition results in MPNST cells 
Table 2 Recommended minimal clinical dataset

Demographics

Date:

Sex

Birth

Diagnosis of NF1, NF2, or schwannomatosis

Inheritance

Mosaicism

Germline mutation

Clinical status

Status

WHO performance status

Pain

Treatment directed at tumor

NF1

\begin{tabular}{|c|c|}
\hline$\geq 6$ Café-au-lait macules & absent \\
\hline Skin fold freckling & absent \\
\hline Iris Lisch nodules & absent \\
\hline Dermal neurofibromas & absent \\
\hline Subcutaneous nodular neurofibromas & absent \\
\hline Diffuse dermal neurofibromas & absent \\
\hline Spinal neurofibromas & not imaged \\
\hline Plexiform neurofibromas & present \\
\hline Optic glioma & unknown \\
\hline Heart defect & unknown \\
\hline Vascular disease & unknown \\
\hline Puberty onset & prepubertal \\
\hline Stature & $<$ th centile \\
\hline Peripheral neuropathy & unknown \\
\hline Aqueductal stenosis & unknown \\
\hline Long bone dysplasia & unknown \\
\hline Sphenoid dysplasia & unknown \\
\hline Scoliosis & unknown \\
\hline Intellectual disability & unknown \\
\hline
\end{tabular}

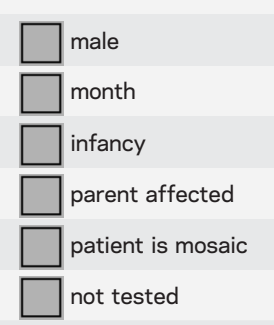

$\begin{array}{ll}\square \text { alive } & \square \text { deceased } \\ \square \text { o } & \square \text { 1 } \\ \square \text { not a problem } & \square \text { occasional } \\ \square \text { no specific therapy } & \square \text { chemotherapy }\end{array}$

$\square$ adolescence $\square$ adulthood $\square$ unknown
$\square$ unknown
$\square$ unknown
$\square$ determined: (specify __)

$\square$ tested + unknown

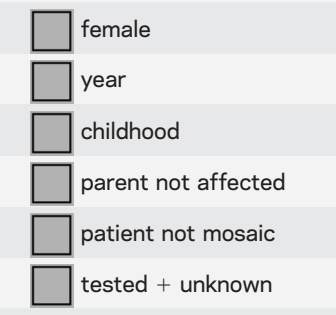

$\square$ determined: (specify _._)

$\square$ present
$\square$ present
$\square$ present
$\square$ scattered
$\square$ scattered
$\square$ scattered
$\square$ absent
$\square$ absent by MRI
$\square$ absent
$\square$ absent
$\square$ absent
$\square$ precocious
$\square$ ath-95th centile
$\square$ absent
$\square$ absent
$\square$ absent
$\square$ absent
$\square$ absent
$\square$ absent

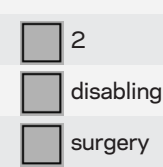

$\square 3$

$\square$ radiation

\section{$\square 4$}

targeted therapy (specify) clinical trial (specify) $\square$ unknown

$\square$ unknown

$\square$ unknown

$\square$ dense

$\square$ dense

$\square$ dense

$\square$ 1-3 levels

$\square$ absent clinically, but no MRI

$\square$ present, asymptomatic

$\square$ present (specify)

$\square$ present (specify)

$\square$ normal

$\square$ >95th centile

$\square$ present

$\square$ present

$\square$ present

$\square$ present

$\square$ present

$\square$ present

$\square$ unknown
$\square$ unknown
$\square$ unknown
$\square$ all levels (cervical, thoracic, lumbar, sacral) $\square$ unknown
$\square$ unknown
$\square$ present, symptomatic, not treated $\square$ present, symptomatic, treated

unknown $\square$ late
$\square$ unknown

Intellectual disability 


\begin{tabular}{|c|c|c|c|c|c|c|}
\hline Continued & & & & & & \\
\hline \multicolumn{7}{|l|}{ NF1 } \\
\hline Learning disability & unknown & absent & present & & & \\
\hline Attention deficit disorder & unknown & absent & present & & & \\
\hline Pheochromocytoma & unknown & absent & present & & & \\
\hline Glomus tumor & unknown & absent & present & & & \\
\hline MPNST & unknown & absent & present & & & \\
\hline Glioma (not optic glioma) & unknown & ]absent & ]present & & & \\
\hline GIST & unknown & $\square$ absent & present & & & \\
\hline Leukemia & unknown & absent & present & & & \\
\hline Breast cancer & unknown & absent & present & & & \\
\hline Other tumors & unknown & $\square$ absent & ]present (specify _._. & & & \\
\hline \multicolumn{7}{|l|}{ NF2 } \\
\hline Vestibular schwannoma & & MRI not done & absent by MRI & unilateral & & unknown \\
\hline Meningioma & & MRI not done & absent by MRI & multiple & & ] unknown \\
\hline Glioma/ependymoma & & MRI not done & absent by MRI & unknown & & \\
\hline Spinal schwannoma & & MRI not done & absent by MRI & multiple & & unknown \\
\hline Dermal schwannoma & & Junknown & absent & present & & \\
\hline Nonvestibular cranial schwannoma & & MRI not done & absent by MRI & present (specify) & & \\
\hline Lenticular opacity & & unknown & absent & present & & \\
\hline \multicolumn{7}{|l|}{ Schwannomatosis } \\
\hline Schwannomas (nonvestibular) & & only by imaging evidence & 1 pathologically confirmed & 2 or more, at least 1 pathologically confirmed & ] unknown & \\
\hline Number of schwannomas & & single & $\square$ scattered & ]dense & Junknown & \\
\hline Vestibular schwannomas & & not imaged & absent by imaging & unilateral & bilateral & ] unknown \\
\hline Meningiomas & & not imaged & absent by imaging & ] single & multiple & ] unknown \\
\hline Other schwannomatosis-related tun & specify) & not investigated & absent & present & unknown & \\
\hline
\end{tabular}

Abbreviations: GIST = gastrointestinal stromal tumors; MPNST = malignant peripheral nerve sheath tumors; NF1 = neurofibromatosis 1 ; NF2 = neurofibromatosis 2 . 
exiting the cell cycle with cessation of growth. ${ }^{18}$ The copy number and expression of the gene product $H M M R$ was found to be a functional marker of responsiveness to aurora kinase inhibition. A variety of less well-defined and reproducible prognosticators are summarized in a review by Farid et al. ${ }^{19}$

- Recently, gene expression analysis of 30 MPNSTs and subsequent immunohistochemical staining of 64 MPNSTs revealed a composite set of survivin (BIRC5), thymidine kinase 1 (TK1), and topoisomerase 2- $\alpha$ (TOP2A) immunohistochemical staining, which stratifies MPNST into high and low risk ${ }^{20}$ independent of age, sex, tumor size, grade, NF1 status, and surgical remission.

- Blakeley et al. ${ }^{21}$ have detected lower blood circulating levels of hepatocyte growth factor in patients with NF2 who responded to bevacizumab. This is the first evidence that a blood biomarker could be used predictively to stratify patients with NF2 ( $\mathrm{n}=$ 13) with the highest likelihood of treatment benefit.

RECOMMENDATIONS FOR SAMPLE COLLECTION AND METHODOLOGY The REiNS biomarker group discussed potential barriers to biomarker research for NF. These barriers include the low prevalence of NF1, NF2, and schwannomatosis, which makes coordinated studies technically difficult and expensive; the extreme variability of these conditions requires expert clinical researchers to accurately phenotype patients. Based on this discussion, the group endorsed the following goals to advance the study of biomarkers within the NF community:

1. Build a prospective biorepository of curated samples. The aim would be to collect longitudinal samples from each patient to facilitate the development of early detection and prognostic markers.

2. Standardize tissue collection at participating institutions. The aim would be to collect all tissues using an identical protocol that meets standards set forth by the American Association for Cancer Research-Food and Drug Administration-National Cancer Institute Cancer Biomarker Collaborative ${ }^{4}$ and would be linked via a shared, anonymized registry (on a Web site). Participating sites would share common consent, SOPs, quality control (see supplemental data on the Neurology ${ }^{\circledR}$ Web site at Neurology.org), minimal clinical dataset, and database.

3. Annotate samples with an agreed minimal clinical dataset. The goal is to link anonymously the phenotypic data to deidentified samples in the biorepository. A proposed minimal clinical dataset developed at a consensus meeting in October 2014 is shown in table 2 . The group anticipates that modifications of this dataset may be required in the future to optimize the utility of biomarker research.
4. Incorporate the decentralized biorepository into existing biorepositories that are used for diagnostic purposes. The biomaterial could thus be used for both diagnosis and research and is not necessarily restricted to an upfront definition of the amount of surplus tissue. Patient care takes preference when allocating the amount of samples used for biomarker investigations.

5. Provide open access to deposited biomarkers to facilitate research. The aim would be to have samples and data open to all qualified researchers with approval of an institutional review board. A biorepository council would govern database requests.

6. An operational and an executive committee will govern requests and audit implementation of SOPs and quality control measures.

7. Incorporate biomarker collection into clinical studies. When feasible, sample collection should be incorporated into prospective clinical trials and natural history studies to help develop pharmacodynamic and predictive biomarkers.

8. Incorporate biomarker collection into routine clinical visits. Patients receiving routine care should be invited to participate in prospective sample collection during routine clinic visits.

\section{RECOMMENDATIONS FOR BIOMARKERS BASED ON EXISTING DATA}

1. Validate individual biomarkers as well as cocktails/ signatures of biomarkers.

2. For ongoing and planned NF trials, studies of drug metabolism/pharmacodynamic biomarkers should be drug-specific.

3. For malignant tumors such as MPNST, explore and validate cDNA, cRNA, and circulating tumor cells as biomarkers.

4. Validate use of extracellular vesicles (exosomes) based on encouraging preliminary data as biomarkers of cancer. $^{22}$

5. Using candidate approach, focus on the clinically relevant questions, i.e., total tumor load, presence of plexiform neurofibroma, evidence of malignant transformation, and taking into account the statistical significance in published studies. We recommend validating the following biomarker candidates in patients with NF1: BIRC5/TK1/TOP2A immunohistochemistry, $\mathrm{ADM}$, interferon- $\gamma$, IGFBP-1, and sAXI.

6. Complement candidate biomarker approach with systematic unbiased approach. Encourage well-powered studies using systematic unbiased approaches, including genomics (DNA, RNA, miRNA next-generation sequencing), metabolomics, and proteomics; that is, further screening with metabolomics, proteomics, expression arrays, and miRNA. 


\section{AUTHOR CONTRIBUTIONS}

C.O. Hanemann: drafting the manuscript, study concept, interpretation of data, development of recommendations and SOPs. Jaishri Blakeley: study concept, review of manuscript, interpretation of data. Fabio Nunes: study concept, review of the manuscript, development of recommendations, development of SOPs. Kent Robertson: study concept, review of manuscript, development of SOPs. Anat Stemmer-Rachamimov: study concept, review of manuscript, development of SOPs. Victor Mautner: study concept, review of manuscript. Andreas Kurtz: study concept, review of manuscript, development of SOPs. Michael Ferguson: study concept, review of manuscript, development of SOPs. Brigitte C. Widemann: study concept, review of manuscript. Gareth Evans: study concept, review of manuscript. Rosalie Ferner: review of manuscript, interpretation of data. Steven L. Carroll: drafting of manuscript, development of autopsy, surgical pathology, and blood biobanking protocols. Bruce Korf: interpretation of data, reviewing manuscript, drafting minimal clinical dataset. Pierre Wolkenstein: review of manuscript. Pam Knight: study concept, review of manuscript. Scott Plotkin: study concept, drafting of manuscript, review of manuscript.

\section{ACKNOWLEDGMENT}

The authors thank Children's Tumor Foundation (CTF) for support and Xandra O. Breakefield, Massachusetts General Hospital, for advising on the manuscript.

\section{STUDY FUNDING}

No targeted funding reported.

\section{DISCLOSURE}

O. Hanemann receives support from Dr. Hadwen Trust and Brain Tumour Research. J. Blakeley has received research support from GlaxoSmithKline, Sanofi-Aventis, and Lily Pharmaceuticals. She has consulted for Abbvie. F. Nunes receives support from Eli Lilly and Company. K. Robertson receives research support from Novartis, NIH, Department of Defense, and Leukemia Lymphoma Society Translational Research Program, and serves on a medical advisory board for General BioTechnologyCook Biomedical. A. Stemmer-Rachamimov receives support from Children's Tumor Foundation, Dana Farber Cancer Institute, and NIH, and serves on the editorial board of Journal of Clinical Pathology. V. Mautner reports no disclosures relevant to the manuscript. A. Kurtz receives support from Seoul National University, College of Veterinary Medicine, Novartis, and Nolitolib, and serves on the editorial board of International Journal of Stem Cells. M. Ferguson serves as an assistant professor with Indiana University. B. Widemann receives research support through NCI CCR Intramural Research Program. G. Evans received support from AstraZeneca and has served on the board of Familial Cancer Journal. R. Ferner receives funds from the Children's Tumor Foundation, European Neurofibromatosis Association, and Springer Publishing Group. S. Carrol received funding from the Department of Defense, NIH/National Cancer Institute, NIH/NINDS, and Children's Tumor Foundation. He serves on the editorial boards of Journal of Neuropathology and Experimental Neurology, Neuro-Oncology, The American Journal of Pathology, and Brain Research Bulletin. B. Korf receives funding from NIH, Department of Defense, Children's Tumor Foundation, Tuberous Sclerosis Alliance, and March of Dimes. He serves on the board of Novartis, Accolade, and Children's Tumor Foundation Medical Advisory Committee, and advises for the Neurofibromatosis Therapeutic Acceleration Project. He has served as editor for Current Protocols in Human Genetics and Annual Reviews of Genetics and Genomics. He has received funding from the American College of Medical Genetics and Genomics, American Academy of Pediatrics, Cambridge HealthTech Institute, Illumina, and Sequenome. He has received publishing royalties from Wiley Blackwell and Elsevier. P. Wolkenstrein serves on the advisory board of Abbvie and Pierre Fabre Dermatologie and consults for Laboratoire d' Uriage and Laboratoire Expansciences. He receives research support from Novartis and the French Government (PHRC 2010). P. Knight reports no disclosures relevant to the manuscript. S. Plotkin receives research support from Children's Tumor Foundation, NIH, and the Department of Defense Neurofibromatosis Clinical Trials Consortium. Go to Neurology.org for full disclosures.

Received November 17, 2015. Accepted in final form March 30, 2016.

\section{REFERENCES}

1. Plotkin SR, Stemmer-Rachamimov AO, Barker FG, et al. Hearing improvement after bevacizumab in patients with neurofibromatosis type 2. N Engl J Med 2009;361:358-367.

2. Plotkin SR, Blakeley JO, Dombi E, et al. Achieving consensus for clinical trials: the REiNS International Collaboration. Neurology 2013;81:S1-S5.

3. Dombi E, Ardern-Holmes SL, Babovic-Vuksanovic D, et al. Recommendations for imaging tumor response in neurofibromatosis clinical trials. Neurology 2013;81: S33-S40.

4. Khleif SN, Doroshow JH, Hait WN; AACR-FDA-NCI Cancer Biomarkers Collaborative. AACR-FDA-NCI Cancer Biomarkers Collaborative consensus report: advancing the use of biomarkers in cancer drug development. Clin Cancer Res 2010;16:3299-3318.

5. Ferner RE. Neurofibromatosis 1 and neurofibromatosis 2: a twenty first century perspective. Lancet Neurol 2007;6:340-351.

6. Lloyd SK, Evans DG. Neurofibromatosis type 2 (NF2): diagnosis and management. Handb Clin Neurol 2013; 115:957-967.

7. Ferner RE, Huson SM, Thomas N, et al. Guidelines for the diagnosis and management of individuals with neurofibromatosis 1. J Med Genet 2007;44:81-88.

8. Listernick R, Ferner RE, Liu GT, Gutmann DH. Optic pathway gliomas in neurofibromatosis-1: controversies and recommendations. Ann Neurol 2007;61:189-198.

9. Kolanczyk M, Mautner V, Kossler N, et al. MIA is a potential biomarker for tumour load in neurofibromatosis type 1. BMC Med 2011;9:82.

10. Yoshida Y, Furumura M, Tahira M, Horie T, Yamamoto O. Serum biomarker in neurofibromatosis type 1. J Dermatol Sci 2012;67:155-158.

11. Hummel TR, Jessen WJ, Miller SJ, et al. Gene expression analysis identifies potential biomarkers of neurofibromatosis type 1 including adrenomedullin. Clin Cancer Res 2010;16: 5048-5057.

12. Jensen $\mathrm{CH}$, Schroder HD, Teisner B, Laursen I, Brandrup F, Rasmussen HB. Fetal antigen 1, a member of the epidermal growth factor superfamily, in neurofibromas and serum from patients with neurofibromatosis type 1. Br J Dermatol 1999;140:1054-1059.

13. Johansson G, Peng PC, Huang PY, et al. Soluble AXL: a possible circulating biomarker for neurofibromatosis type 1 related tumor burden. PLoS One 2014;9:e115916.

14. Park SJ, Sawitzki B, Kluwe L, Mautner VF, Holtkamp N, Kurtz A. Serum biomarkers for neurofibromatosis type 1 and early detection of malignant peripheral nerve-sheath tumors. BMC Med 2013;11:109.

15. Danielsen SA, Lind GE, Kolberg M, et al. Methylated RASSF1A in malignant peripheral nerve sheath tumors identifies neurofibromatosis type 1 patients with inferior prognosis. Neuro Oncol 2015;17:63-69.

16. Gong M, Ma J, Li M, Zhou M, Hock JM, Yu X. MicroRNA-204 critically regulates carcinogenesis in malignant peripheral nerve sheath tumors. Neuro Oncol 2012;14:1007-1017.

17. Yu J, Deshmukh H, Payton JE, et al. Array-based comparative genomic hybridization identifies CDK4 and FOXM1 alterations as independent predictors of survival in malignant peripheral nerve sheath tumor. Clin Cancer Res 2011;17:1924-1934. 
18. Mohan P, Castellsague J, Jiang J, et al. Genomic imbalance of HMMR/RHAMM regulates the sensitivity and response of malignant peripheral nerve sheath tumour cells to aurora kinase inhibition. Oncotarget 2013;4:80-93.

19. Farid M, Demicco EG, Garcia R, et al. Malignant peripheral nerve sheath tumors. Oncologist 2014;19: 193-201.

20. Kolberg M, Holand M, Lind GE, et al. Protein expression of BIRC5, TK1, and TOP2A in malignant peripheral nerve sheath tumours: a prognostic test after surgical resection. Mol Oncol 2015;9:1129-1139.

21. Blakeley JO, Ye X, Duda DG, et al. Efficacy and biomarker study of bevacizumab for hearing loss resulting from neurofibromatosis type 2-associated vestibular schwannomas. J Clin Oncol 2016;34:1669-1675.

22. Kros JM, Mustafa DM, Dekker LJ, Sillevis Smitt PA, Luider TM, Zheng PP. Circulating glioma biomarkers. Neuro Oncol 2015;17:343-360. 


\section{Neurology}

\section{Current status and recommendations for biomarkers and biobanking in neurofibromatosis \\ C. Oliver Hanemann, Jaishri O. Blakeley, Fabio P. Nunes, et al. Neurology 2016;87;S40-S48 \\ DOI 10.1212/WNL.0000000000002932}

\section{This information is current as of August 15, 2016}

\section{Updated Information \& Services \\ Supplementary Material}

\section{References}

Citations

\section{Subspecialty Collections}

Permissions \& Licensing

Reprints including high resolution figures, can be found at: http://n.neurology.org/content/87/7_Supplement_1/S40.full

Supplementary material can be found at: http://n.neurology.org/content/suppl/2016/08/15/WNL.0000000000002 932.DC1

http://n.neurology.org/content/suppl/2016/08/15/WNL.0000000000002 932.DC2

This article cites 22 articles, 6 of which you can access for free at: http://n.neurology.org/content/87/7_Supplement_1/S40.full\#ref-list-1

This article has been cited by 2 HighWire-hosted articles: http://n.neurology.org/content/87/7_Supplement_1/S40.full\#\#otherartic les

This article, along with others on similar topics, appears in the following collection(s):

\section{Neurofibromatosis}

http://n.neurology.org/cgi/collection/neurofibromatosis

Information about reproducing this article in parts (figures,tables) or in its entirety can be found online at:

http://www.neurology.org/about/about_the_journal\#permissions

Information about ordering reprints can be found online:

http://n.neurology.org/subscribers/advertise

Neurology ${ }^{\circledR}$ is the official journal of the American Academy of Neurology. Published continuously since 1951, it is now a weekly with 48 issues per year. Copyright @ 2016 American Academy of Neurology. All rights reserved. Print ISSN: 0028-3878. Online ISSN: 1526-632X.

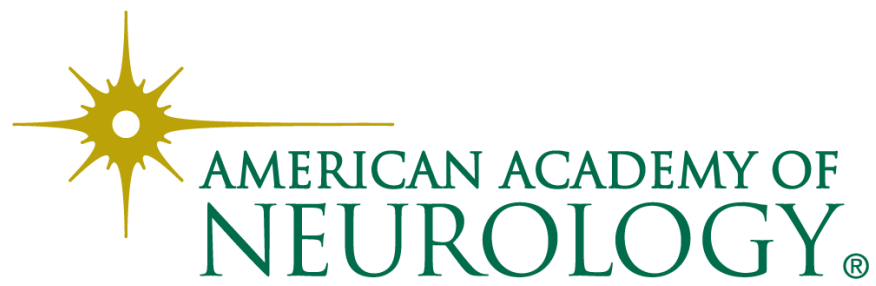

